University of New Hampshire

University of New Hampshire Scholars' Repository

Space Science Center

Institute for the Study of Earth, Oceans, and

Space (EOS)

1998

\title{
SONTRAC-A low background, large area solar neutron spectrometer
}

James M. Ryan

University of New Hampshire, James.Ryan@unh.edu

D Holslin

Science Applications International Corporation

John R. Macri

University of New Hampshire - Main Campus, John.Macri@unh.edu

Mark L. McConnell

University of New Hampshire - Main Campus, mark.mcconnell@unh.edu

Cornelia B. Wunderer

University of California - Berkeley

Follow this and additional works at: https://scholars.unh.edu/ssc

Part of the Astrophysics and Astronomy Commons

\section{Recommended Citation}

SONTRAC-A low background, large area solar neutron spectrometer Ryan, J. M. and Holslin, D. and Macri, J. R. and McConnell, M. L. and Wunderer, C. B., AIP Conference Proceedings, 450, $592-597$ (1998), DOI:http://dx.doi.org/10.1063/1.56963

This Conference Proceeding is brought to you for free and open access by the Institute for the Study of Earth, Oceans, and Space (EOS) at University of New Hampshire Scholars' Repository. It has been accepted for inclusion in Space Science Center by an authorized administrator of University of New Hampshire Scholars' Repository. For more information, please contact Scholarly.Communication@unh.edu. 


\section{AIP | Proceedings}

\section{SONTRAC-A low background, large area solar neutron spectrometer}

J. M. Ryan, D. Holslin, J. R. Macri, M. L. McConnell, and C. B. Wunderer

Citation: AIP Conference Proceedings 450, 592 (1998); doi: 10.1063/1.56963

View online: http://dx.doi.org/10.1063/1.56963

View Table of Contents:

http://scitation.aip.org/content/aip/proceeding/aipcp/450?ver=pdfcov

Published by the AIP Publishing

\section{Articles you may be interested in}

Development of multichannel low-energy neutron spectrometera)

Rev. Sci. Instrum. 85, 11E125 (2014); 10.1063/1.4895826

Light output function and assembly of the time-of-flight enhanced diagnostics neutron spectrometer plastic scintillators for background reduction by double kinematic selection at EASTa)

Rev. Sci. Instrum. 85, 11E112 (2014); 10.1063/1.4886762

A neutron spectrometer for precise measurements of DT neutrons from 10 to 18 $\mathrm{MeV}$ at OMEGA and the National Ignition Facility

Rev. Sci. Instrum. 72, 854 (2001); 10.1063/1.1323243

A sensitive neutron spectrometer for the National Ignition Facility

Rev. Sci. Instrum. 72, 846 (2001); 10.1063/1.1323242

Distinction between DD and DT neutrons in the TFTR neutron collimator (abstract) Rev. Sci. Instrum. 68, 552 (1997); 10.1063/1.1147650 


\title{
SONTRAC-A Low Background, Large Area Solar Neutron Spectrometer
}

\author{
J.M. Ryan ${ }^{1}$, D. Holslin' ${ }^{2}$ J.R. Macri' ${ }^{1}$, M.L. McConnel1 ${ }^{1}$, C.B. Wunderer ${ }^{1}$ \\ ${ }^{1}$ Space Science Center, University of New Hampshire, Durham, NH 03824 \\ ${ }^{2}$ Science Applications International Corporation, San Diego, CA 92121
}

\begin{abstract}
SONTRAC is a scintillating fiber neutron detector designed to measure solar flare neutrons from a balloon or spacecraft platform. The instrument is comprised of alternating orthogonal planes of scintillator fibers viewed by photomultiplier tubes and image intensifier/CCD camera optics. It operates by tracking the paths of recoil protons from the double scatter of 20 to $200 \mathrm{MeV}$ neutrons off hydrogen in the plastic scintillator, thereby providing the necessary information to determine the incident neutron direction and energy. SONTRAC is also capable of detecting and measuring high-energy gamma rays $>20 \mathrm{MeV}$ as a "solid-state spark chamber." The self-triggering and track imaging features of a prototype for tracking in two dimensions have been demonstrated in calibrations with cosmic-ray muons, 14 to $\sim 65 \mathrm{MeV}$ neutrons and $\sim 20 \mathrm{MeV}$ protons.
\end{abstract}

\section{INTRODUCTION AND MOTIVATION}

Neutrons above the nuclear binding energy are ubiquitous in cosmic ray interactions, whether those reactions occur on the surface of the Sun or in the earth's atmosphere. These secondary neutrons are difficult to measure because they lack charge, and thus, do not interact readily with detector material.

When high-energy charged particle reactions occur on the surface of the Sun, neutrons carry away information about the spectrum of ions that produced them. They can be used as diagnostic measures of the accelerated ion spectrum in solar flares $(1,2)$. As illustrated in Fig. 1, gamma rays are produced efficiently by protons below $20 \mathrm{MeV}$ and above $300 \mathrm{MeV}$. The intervening region of the proton spectrum is most effective in producing neutrons of comparable energy. To determine the proton spectrum in this region secondary neutrons must be measured. This measurement must take place from a space platform or a balloon. In either environment the background is high making the measurement difficult.

To perform these measurements neutron telescopes based on double scatters have been used (3-6). Because of its directional properties a neutron telescope one can reject 
much of the background, thereby enhancing the signal-to-noise ratio, making these instruments effective in high background environments. Such instruments have applications in other disciplines including atmospheric physics, radiation therapy and nuclear materials monitoring.

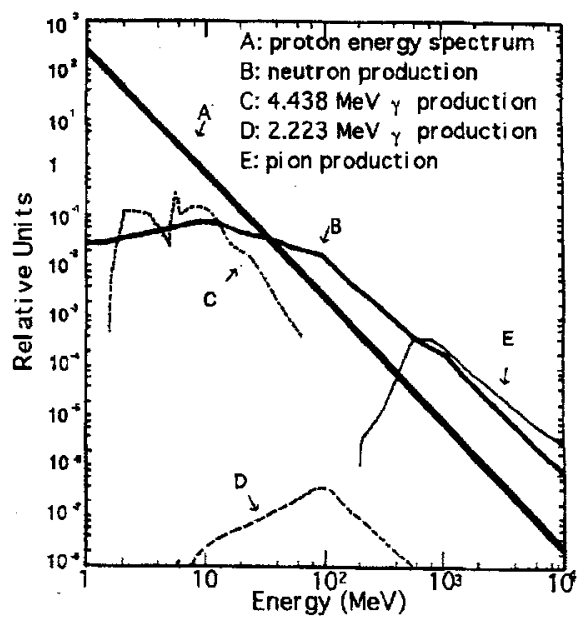

FIGURE 1. Spectrum-weighted emissions of solar flare gamma rays and neutrons.

\section{TRACKING DETECTOR CONCEPT}

We have been studying a tracking detector design for measuring neutrons in the difficult range of 20 to $250 \mathrm{MeV}$. The design is based around a closely packed bundle of square cross section plastic-scintillator fibers. The fibers are arranged in stacked planes with the fibers in each plane orthogonal to those in adjacent planes. This configuration allows one to record stereoscopic images of ionization tracks.

Neutrons undergo elastic scattering off hydrogen within the organic plastic-scintillator fibers, scattering at right angles with respect to the scattered proton at non-relativistic energies. The Bragg peak (greater ionization at the end of the track) is used to determine proton track direction. A second proton scatter of the neutron provides the spatial information that is necessary and sufficient to determine the incident neutron energy and direction. An image and spectrum of the neutron source can be constructed from these data. The angular and energy resolution of the instrument depend upon our ability to track the recoil protons and measure the scintillation light.

A functional diagram of an experiment utilizing the SONTRAC concept is shown in Fig. 2. The detector's spectroscopic, track detection and imaging components cover the entire light-emitting area of the fiber bundle and are duplicated in the orthogonal dimension (not shown). Scintillation light signal is collected and processed at both ends of the fiber bundle. At one end a signal above threshold from a photomultiplier tube (PMT) gates the trigger logic circuitry. At the other end, fiber-optic tapers and a pair of 
image intensifiers demagnify, capture and hold the scintillation-light image of the track(s) for the CCD camera. The first image intensifier is always ON, holding the image for $\sim 1 \mathrm{~ms}$. The second image intensifier is normally in the gated-OFF condition. However, with the particle coincidence requirements satisfied, the track image and PMT pulse height data are acquired, combined with any auxiliary data and recorded.

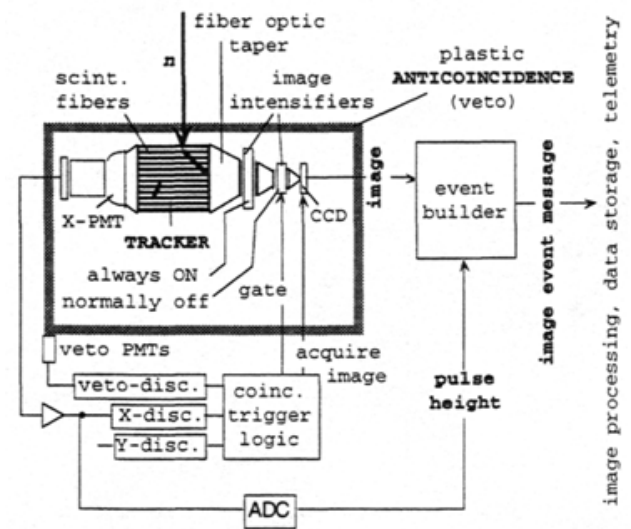

FIGURE 2. Complete instrument functional and logic diagram.

A $4 \pi$ plastic scintillator shield surrounds the tracker to reject incoming cosmic rays. Other charged particles produced in the detectors cannot escape the detector volumewithout being registered. They represent lost energy and can be rejected.

The initial design of the SONTRAC instrument suffered at the time from a lack of affordable technology and existed in simulations only (7-9). Due to advancements in technology, we have been able to construct a simple prototype of the instrument to verify the concept and identify any fundamental technical limitations.

Fig. 3 is a schematic of the SONTRAC prototype operating at UNH. The prototype is a $10 \mathrm{~cm}$ long bundle of $250 \mu \mathrm{m}$-square $(230 \mu \mathrm{m}$ active), multiclad, organic, scintillating, plastic fibers (Bicron BCF-99-55) within a $12.7 \mathrm{~mm}$ square envelope. It is, therefore, limited to tracking in two dimensions. The fiber thickness was chosen such that a $10 \mathrm{MeV}$ recoil proton traverses several fibers. The fiber pitch is $300 \mu \mathrm{m}$ (including cladding and EMA) and the calculated range of a $10 \mathrm{MeV}$ proton (50\% of the proposed neutron threshold energy) is $1.25 \mathrm{~mm}(\sim 4$ fibers $)$. The PMT is a bialkali photocathode device from Thorn EMI. Two $18 \mathrm{~mm}$ diameter, single-MCP, generation-2 image intensifiers from DEP are employed. The S20 photocathode for the first image intensifier was selected for its response to scintillation light. The P43 phosphor holds the image for $\sim 1 \mathrm{~ms}$. The second intensifier's photocathode (S25) and phosphor (P43) provide good spectral matches to the output of the first intensifier and the input to the CCD sensor, respectively. The CCD camera (Pulnix TM-9701) is an inexpensive, progressive scanning camera with digital readout and control, asynchronous external trigger and full frame shutter capability. The Matrox frame grabber and image processor operate within a Pentium PC. 


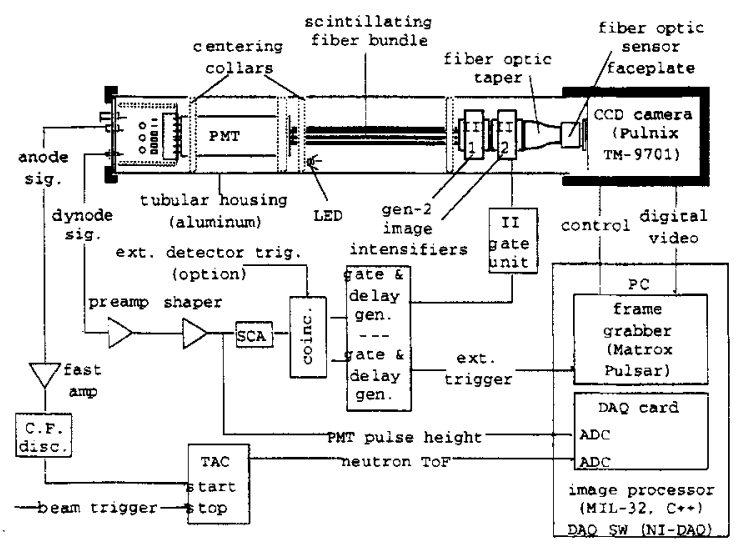

FIGURE 3. Prototype functional and logic diagram.

\section{PROTOTYPE PERFORMANCE AND FLIGHT DESIGN}

Tracks of recoil protons near our trigger threshold energy $(14 \mathrm{MeV})$ have been published elsewhere (10). These data show that protons as low as $\sim 10 \mathrm{MeV}$ can be tracked over 4 or 5 fibers with a clear Bragg peak. The scintillation light also conforms to the expected distribution (within errors) of the ionization rate.

The SONTRAC prototype was also exposed to $20 \mathrm{MeV}$ and $27 \mathrm{MeV}$ neutrons and to $\sim 20 \mathrm{MeV}$ protons at the Crocker Nuclear Laboratory at the University of California at Davis. Additional measurements were made with $\sim 65 \mathrm{MeV}$ neutrons, although it was not possible to get a clean beam in the time available. Fig. 4 shows two CCD images of neutron interactions in the prototype fiber bundle. On the left is a double scatter event displaying two recoil proton tracks from a neutron $(\sim 65 \mathrm{MeV})$ incident from the top of the figure. On the right is a single proton recoil track from a $28 \mathrm{MeV}$ neutron incident from the top left of the figure. The individual fibers are seen in the ionization tracks. Note that the Bragg peak is evident establishing the proton recoil direction. Gaps in the track images are due the ionizing particles traversing the passive cladding and EMA materials.
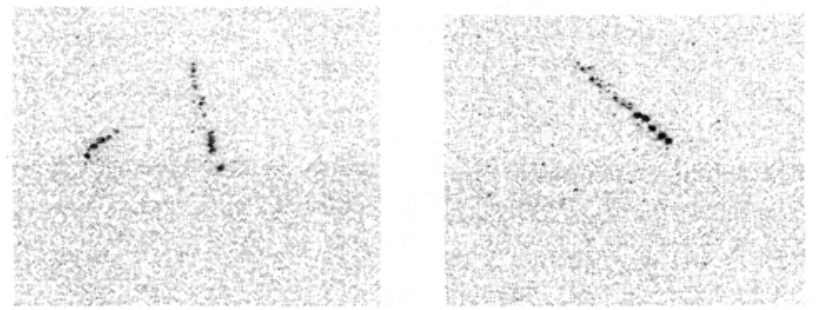

FIGURE 4. Double scatter of a $\sim 65 \mathrm{MeV}$ neutron (left) and a $28 \mathrm{MeV}$ proton (right).

Fig. 5 is a cosmic-ray muon image recorded with the SONTRAC prototype, demonstrating the detector's ability to track minimum-ionizing particles. (The fiber 
mask is shown for reference.) This is necessary to track conversion electrons in high energy gamma detectors. We calculate that $\sim 4$ photoelectrons/fiber are generated for amplification within the image intensifier.

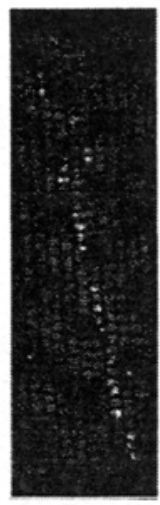

FIGURE 5. A cosmic-ray muon track.

A sketch of a larger volume detector for particle tracking in three dimensions is shown in Fig. 6. It employs orthogonal layers of scintillating fibers in a $10 \times 10 \times 10$ $\mathrm{cm}$ bundle with one PMT (left) and one image intensifier/CCD chain (right) in each dimension. The fiber block would be surrounded by charged particle detectors to (1) reject cosmic ray protons and electrons and (2) to detect the escape of secondary charged particles from internal reactions. The flight instrument would occupy a space less than $50 \times 50 \times 20 \mathrm{~cm}^{3}$ with a total mass less than $26 \mathrm{~kg}$. We estimate that the proposed instrument will have an effective area for detecting and measuring 15 to $60 \mathrm{MeV}$ neutrons of $\sim 2 \mathrm{~cm}^{2}$, approximately the same as that of the imaging Compton telescope, COMPTEL, a $1460 \mathrm{~kg}$ instrument (6).

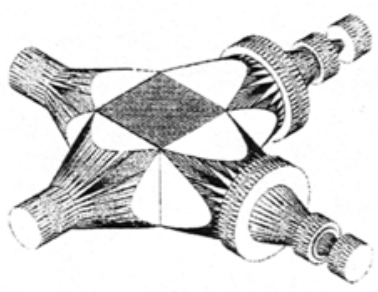

FIGURE 6. Sketch of a $10 \times 10 \times 10 \mathrm{~cm}$ 3-d imager.

The typical energy resolution is on the order of $10 \%$ or better for the majority of neutron events. The angular resolution is largely determined by the pitch of the fibers, i.e., the uncertainty in the end points of the tracks. For a $45^{\circ}$ scatter and $300 \mu \mathrm{m}$ fiber 
pitch it ranges from $23^{\circ}$ at $20 \mathrm{MeV}$ to $5^{\circ}$ at $50 \mathrm{MeV}$ to $0.7^{\circ}$ at $200 \mathrm{MeV}$. This angular resolution is the basis for our high signal-to-noise ratio and thus our good sensitivity.

\section{CONCLUSIONS}

We have demonstrated the important features of the SONTRAC instrument with a laboratory prototype. This will help to determine the engineering parameters important for the SONTRAC application (e.g., scintillating fiber pitch, light yields, gains, photocathode and phosphor selection, gating delays and intervals). Although the prototype is limited to tracking in two dimensions, we obtained self-triggered images of tracks of $\sim 20 \mathrm{MeV}$ protons, recoil protons from 14 to $\sim 65 \mathrm{MeV}$ neutrons and minimum ionizing tracks of cosmic ray muons. The track images and the associated pulse height information provide good resolution measurements of both the direction and energy of the incident radiation. An extension to 3-dimensional tracking promises to provide unprecedented measurement capabilities for studies in solar physics and other fields.

\section{ACKNOWLEDGMENTS}

We wish to thank Prof. Patrick Papin and David Smith for their assistance with the $14 \mathrm{MeV}$ neutron calibration. We also wish to thank Drs. Carlos Castaneda and Juan Romero with the high energy neutron and proton calibrations and instrument simulations. We also thank Mr. Tim O'Gorman for simulations and Drs. Glenn Frye and Tom Jenkins for their support. This work is supported under NASA's Space Physics Supporting Research and Technology program.

\section{REFERENCES}

1. H. Hudson and J. Ryan, Annual Review Astronomy and Astrophysics 33, 239-82 (1995).

2. W. T. Vestrand and J. A. Miller, "Particle Acceleration During Solar Flares," in The Many Faces of the Sun: The Scientific Results of the Solar Maximum Mission, edited by B. Haisch et al. (Springer Verlag, New York, 1998), in press.

3. A. M. Preszler, G. M. Simnett and R. S. White, J. Geophys. Res. 79, 17-22 (1974).

4. G. Kanbach, C. Reppin and V. Schönfelder, J. Geophys, Res. 79, 5159-265 (1974).

5. J. A. Lockwood, C. Chen, L. A. Friling and R. N. St. Onge, J. Geophys. Res. 81, 6211-6216 (1976).

6. J. M. Ryan et al., "COMPTEL as a Solar Gamma Ray and Neutron Detector," in Data Analysis in Astronomy, Plenum Press: New York, (1992), pp. 261-270.

7. G. M. Frye, T. L. Jenkins and A. Owens, "SONTRAC: A Solar Neutron Track Chamber Detector," in 19th ICRC, 5, (1985), pp. 498-501.

8. G. M. Frye, C. J. Hall, T. L. Jenkins and G. N. Pendleton, "Predicted Performance of Solar Neutron Track Chamber Detector (SONTRAC)," in 20th ICRC, 4, (1987), pp. 392-394.

9. G. N. Pendleton, "Predicted performance of a prototype solar neutron detector," Ph.D. Thesis, Case Western Reserve Univ. (1988).

10. J. M. Ryan et al., "A Prototype for SONTRAC, a Scintillating Plastic Fiber Detector for Solar Neutron Spectroscopy," in SPIE, 3114, (1997), pp. 514-525. 